\title{
Linearized Homotopy Perturbation Method for Two Nonlinear Problems of Duffing Equations
}

\author{
Chein-Shan Liu \\ Correspondence: Center of Excellence for Ocean Engineering, Center of Excellence for the Oceans, National Taiwan \\ Ocean University, Keelung 20224, Taiwan
}

\author{
Received: September 19, 2021 Accepted: October 21, 2021 Online Published: October 27, 2021 \\ doi:10.5539/jmr.v13n6p10 URL: https://doi.org/10.5539/jmr.v13n6p10
}

\begin{abstract}
In the paper, we solve two nonlinear problems related to the Duffing equations in space and in time. The first problem is the bifurcation of Duffing equation in space, wherein a critical value of the parameter initiates the bifurcation from a trivial solution to a non-trivial solution. The second problem is an unconventional periodic problem of Duffing equation in time to determine period and periodic solution. To save computational cost and even enhance the accuracy in seeking higher order analytic solutions of these two problems, a modified homotopy perturbation method is invoked after a linearization technique being exerted on the Duffing equation, whose nonlinear cubic term is decomposed at two sides via a weight factor, such that the Duffing equation is linearized as the Mathieu type differential equation. The constant preceding the displacement is expanded in powers of homotopy parameter and the coefficients are determined to avoid secular solutions appeared in the derived sequence of linear differential equations. Consequently, after setting the homotopy parameter equal to unity and solving the amplitude equation, the higher order bifurcated solutions can be derived explicitly. For the second problem, we can determine the period and periodic solution in closed-form, which are very accurate. For the sake of comparison the results obtained from the fourth-order Runge-Kutta numerical method are used to evaluate the presented analytic solutions.
\end{abstract}

Keywords: Nonlinear Duffing equation, Analytic solution, Mathieu equation, Linearized homotopy perturbation method

\section{Introduction}

In the last few decades, considerable attention was directed towards the analytic solutions for nonlinear oscillators, since most nonlinear phenomena are modeled by nonlinear differential equations. The determination of period and periodic solution of nonlinear oscillators is one of the most important topics in the research of nonlinear physical problems. Some analytic techniques have been used to treat nonlinear differential equations, like the perturbation methods (Nayfeh, 1979; $\mathrm{He}$, 2005). If one attempts to obtain an approximate analytic solution of nonlinear problem, the functional iteration method, on the other hand, is a convenient tool. An earlier functional iteration method is the Picard iteration method to find approximate solutions for nonlinear differential equations. However, the Picard iteration has a major drawback with slow convergence. To accelerate the convergence, He $(1999,2000 \mathrm{a})$ has proposed a variational iteration method for solving strongly nonlinear problems (Herisanu \& Marinca, 2010).

There are many computational methods to determine the periodic solutions of nonlinear oscillators, for example, the harmonic balance method (Donescu, Virgin \& Wu, 1996; Wu, Sun \& Lim, 2006; Liu, Thomas, Dowell, Attar \& Hall, 2006), the variational iteration method (He, 1999; Ozis \& Yildirim, 2007), the homotopy perturbation method (He, 2000b; Shou, 2009), the parameter-expanding method (Koroglu \& Ozis, 2011), the exp-function method (He \& Abdou, 2007), the differential transform method (Chu \& Lo, 2011), and the optimally scaled polynomial-Fourier-series method (Khan, Liu \& Riaz, 2016). The harmonic balance method approximates the periodic solutions of nonlinear oscillators expanded in terms of the Fourier series, and the terms associated with each harmonic component are balanced. In general, this process could be very complicated when the order increases.

The parameter-expanding method developed by He $(2001,2006)$ is a convenient method for nonlinear differential equations, which has been shown to effectively, easily and accurately solve a large class of linear and nonlinear problems with components that converge rapidly to accurate solutions. Some papers pertinent to the parameter-expanding method have been developed. The first one was proposed by He (2002) to modify the Lindstedt-Poincare methods for some strongly nonlinear oscillations; Xu (2007) suggested to use He's parameter-expanding methods for strongly nonlinear oscillators, while Tao (2009) proposed a frequency-amplitude relationship of nonlinear oscillator by using He's parameter-expanding method. 
He's homotopy perturbation method (He, 2000b, 2003) is expressing both the analytic solution and an unknown frequency in terms of a homotopy parameter, while unknown coefficients are determined by equating each power of the homotopy parameter. The recent works for the homotopy perturbation method applied to nonlinear oscillators can refer (He \& ElDib, 2020, 2021). In the original He's homotopy perturbation method, the computational cost is grew algebraically when the order is increasing. Inspired by the Newton linearization technique in the development of Newton iteration method, we propose a linearization technique for the nonlinear differential equation around a fundamental solution satisfying the prescribed conditions. Then, He's homotopy perturbation method is applied to the linearized differential equation of Mathieu type to determine the period and periodic solution.

Traditionally, the perturbation method is applied to the original nonlinear differential equation and then derived a sequence of linear differential equations to determine the analytic solutions. Here, we first linearize the nonlinear differential equation to a linear one and then apply the perturbation method to the linearized differential equation. In doing so, the computational cost can be reduced, and the work to seek the period and analytic solution becomes simpler and easier.

The present paper is organized as follows. In Section 2, the Duffing equation to describe the deflection of a short strut in an one-dimensional space is given and we transform it into a Mathieu equation with forcing terms using a novel linearizing technique. The linearized homotopy method is developed in Section 3 to seek the bifurcated solutions of eigenfunction and eigenvalue up to the third-order analytic approximation, whose accuracy with different length of the strut is assessed by comparing to that computed from the fourth-order Runge-Kutta method (RK4). In Section 4, we turn our attention to an unconventional periodic problem of the Duffing equation in time, where the period (or frequency) and mode shape of the free vibration of the Duffing equation are determined from a zero initial displacement and an extra condition of displacement at one quarter of the unknown period. Here, we develop a linearized homotopy method to determine the period and the free vibration mode up to second-order and the accuracy of the period and the analytic solution are confirmed by comparing to the exact one and the one computed from the RK4. Finally, the conclusions are drawn in Section 5 .

\section{Linearizing Duffing Equation to Mathieu Equation}

Consider the deflection of a short strut governed by the Duffing equation:

$$
\begin{aligned}
& w^{\prime \prime}(z)+w(z)-w^{3}(z)=0,0<z<L, \\
& w(0)=w(L)=0,
\end{aligned}
$$

which only has the trivial solution $w(z)=0$ when the length $L$ is shorter than a critical value.

To unfold the bifurcation behavior of Eqs. (1) and (2), Liao (2004) has considered the following transformations:

$$
x:=\frac{\pi z}{L}, \varepsilon=\frac{L^{2}}{\pi^{2}}, v(x)=w(z)
$$

and then they become

$$
\begin{aligned}
& v^{\prime \prime}(x)+\varepsilon\left[v(x)-v^{3}(x)\right]=0,0<x<\pi, \\
& v(0)=v(\pi)=0 .
\end{aligned}
$$

Further letting

$$
y(x):=\frac{v(x)}{A}, A:=v(\pi / 2) \Rightarrow y(\pi / 2)=\frac{v(\pi / 2)}{A}=1,
$$

Eqs. (4) and (5) are recast to

$$
\begin{aligned}
& y^{\prime \prime}(x)+\varepsilon\left[y(x)-A^{2} y^{3}(x)\right]=0,0<x<\pi, \\
& y(0)=0, y(\pi / 2)=1, y(\pi)=0,
\end{aligned}
$$

where $A$ is an unknown amplitude function of $\varepsilon$ to be determined such that the problem possesses a non-trivial solution (He, 2005; Motsa \& Sibanda, 2012). Eqs. (7) and (8) constitute a three-point nonlinear boundary value problem.

In (Kahn \& Zarmi, 1998), the relation between $\varepsilon>0$ and $A$ is given by

$$
\sqrt{\varepsilon}=\frac{2}{\pi} \int_{0}^{A} \frac{d s}{\sqrt{A^{2}-s^{2}-\left(A^{4}-s^{4}\right) / 2}} .
$$


As pointed in (Liao, 2004; He, 2005), the traditional perturbation method led to

$$
A= \pm 2 \sqrt{\frac{\varepsilon-1}{3}},
$$

which is merely applicable in a small range of $\varepsilon>1$.

Before deriving the analytic solution for Eqs. (7) and (8), we discuss the linearization of Eq. (7). For a single nonlinear equation $f(x)=0$, the Newton technique is giving an initial guess $x_{0}$ and solving a linearized equation

$$
f(x)=f\left(x_{0}\right)+f^{\prime}\left(x_{0}\right)\left(x-x_{0}\right) \approx 0
$$

to approximate the solution of $f(x)=0$, which results to the famous Newton iterative method:

$$
x_{n+1}=x_{n}-\frac{f\left(x_{n}\right)}{f^{\prime}\left(x_{n}\right)}, n=0,1, \ldots
$$

To satisfy the three-point conditions in Eq. (8), the zeroth-order fundamental solution is found to be

$$
y_{0}(x)=\sin x .
$$

Similarly, giving a zeroth-order fundamental solution $y_{0}(x)$ in Eq. (13), we can linearize the nonlinear cubic term $y^{3}(x)$ in Eq. (7) around $y_{0}(x)$ by

$$
y^{\prime \prime}(x)+\varepsilon y(x)-\varepsilon A^{2}\left\{y_{0}^{3}(x)+3 y_{0}^{2}(x)\left[y(x)-y_{0}(x)\right]\right\}=0,
$$

which can be rearranged to

$$
y^{\prime \prime}(x)+\varepsilon y(x)-3 \varepsilon A^{2} y_{0}^{2}(x) y(x)=-2 \varepsilon A^{2} y_{0}^{3}(x)
$$

We generalize Eq. (15) as

$$
y^{\prime \prime}(x)+\varepsilon y(x)-q_{0} \varepsilon A^{2} y_{0}^{2}(x) y(x)=\left(1-q_{0}\right) \varepsilon A^{2} y_{0}^{3}(x),
$$

where $q_{0}$ is a weight factor. Taking $q_{0}=3$, Eq. (16) coincides to Eq. (15). Obtained from Eq. (13):

$$
y_{0}^{2}(x)=\frac{1}{2}(1-\cos 2 x), y_{0}^{3}(x)=\frac{1}{4}(3 \sin x-\sin 3 x),
$$

and inserting them into Eq. (16), leads to a linear differential equation:

$$
y^{\prime \prime}(x)+\left(\lambda-2 e_{0} \cos 2 x\right) y(x)=3 e_{1} \sin x-e_{1} \sin 3 x,
$$

where

$$
\lambda=\varepsilon-\frac{q_{0} \varepsilon A^{2}}{2}, e_{0}=-\frac{q_{0} \varepsilon A^{2}}{4}, e_{1}=\frac{\left(1-q_{0}\right) \varepsilon A^{2}}{4}=e_{0}+\frac{\varepsilon A^{2}}{4} .
$$

Eq. (18) is the Mathieu equation with periodic forcing terms appeared on the right-hand side. We are going to develop the homotopy perturbation method [He (2000b)] to seek the higher order analytic solutions of Eqs. (7) and (8) through Eq. (18).

\section{Homotopy Perturbation Method for Forced Mathieu Equation}

Now, we demonstrate how to apply He's homotopy perturbation method [He $(2000 \mathrm{~b}, 2003)]$ for solving the eigenvalue problem of Mathieu equation [Cartmell (1990); Bujurke, Salimath \& Shiralashetti (2008); Gadella, Giacomini \& Lara (2015); Liu (2020)], which is a special case of the following one with $e_{1}=0$ :

$$
\begin{aligned}
& y^{\prime \prime}(x)+\left(\lambda-2 e_{0} \cos 2 x\right) y(x)=3 e_{1} \sin x-e_{1} \sin 3 x, 0<x<\pi, \\
& y(0)=y(\pi)=0,
\end{aligned}
$$

where the eigenvalue $\lambda$ is to be determined.

The first step for the construction of He's homotopy perturbation method [He $(2003,2006)]$ is writing Eq. (20) as a homotopy perturbation equation:

$$
y^{\prime \prime}(x)+\left(\lambda-2 p e_{0} \cos 2 x\right) y(x)=3 p e_{1} \sin x-p e_{1} \sin 3 x, p \in[0,1] .
$$


When $p=1$, Eq. (20) is recovered. Hereon, $p$ can be understood as a bookkeeping parameter to help us correctly deriving the related equations while at the final stage it is set equal to unity.

Then, the eigenfunction and eigenvalue are determined by

$$
\begin{aligned}
& y(x)=y_{0}(x)+\sum_{k=1}^{m} p^{k} y_{k}(x), \\
& \lambda=1-\sum_{k=1}^{m} \alpha_{k} p^{k},
\end{aligned}
$$

where $\alpha_{k}$ and $y_{k}(x), k=1,2, \ldots, m$ are to be determined and $y_{0}(x)$ in Eq. (13) is the zeroth-order solution. Only when $\lambda$ is given by the characteristic Eq. (24), the eigenfunction exists. We can derive the eigenvalue $\lambda$ and then the corresponding eigenfunction in Eq. (23) by inserting $p=1$ and $y_{k}(x), k=0,1,2, \ldots, m$.

Inserting Eqs. (23) and (24) into Eq. (22) and equating the coefficients preceding $p^{k}, k=0,1,2, \ldots, m$, we can derive

$$
\begin{aligned}
& y_{0}^{\prime \prime}(x)+y_{0}(x)=0, \quad y_{0}(0)=0, y_{0}(\pi / 2)=1, y_{0}(\pi)=0, \\
& y_{1}^{\prime \prime}(x)+y_{1}(x)=\left(\alpha_{1}+2 e_{0} \cos 2 x\right) y_{0}+3 e_{1} \sin x-e_{1} \sin 3 x, \quad y_{1}(0)=y_{1}(\pi / 2)=y_{1}(\pi)=0, \\
& y_{k}^{\prime \prime}(x)+y_{k}(x)=\left(\alpha_{1}+2 e_{0} \cos 2 x\right) y_{k-1}+\sum_{i=0}^{k-2} \alpha_{k-i} y_{i}, \quad y_{k}(0)=y_{k}(\pi / 2)=y_{k}(\pi)=0, k=2, \ldots, m .
\end{aligned}
$$

They are sequential linear differential equations, which can be solved exactly by inserting the solutions obtained from the previous steps into the right-hand sides.

Inserting Eq. (13) into Eq. (26), yields

$$
y_{1}^{\prime \prime}(x)+y_{1}(x)=\left(\alpha_{1}+2 e_{0} \cos 2 x\right) \sin x+3 e_{1} \sin x-e_{1} \sin 3 x .
$$

To avoid the secular solution, we have to take

$$
\alpha_{1}=e_{0}-3 e_{1}=\frac{\left(2 q_{0}-3\right) \varepsilon A^{2}}{4} .
$$

Then, the first-order solution obtained from Eq. (28) is

$$
y_{1}(x)=\frac{e_{1}-e_{0}}{8}(\sin x+\sin 3 x)=\frac{\varepsilon A^{2}}{32}(\sin x+\sin 3 x),
$$

where the term $\sin x$ appears to render $y_{1}(\pi / 2)=0$.

Inserting $p=1$ into Eq. (24) with $m=1$ and with the aid of Eqs. (19) and (29), it follows that

$$
\varepsilon-\frac{q_{0} \varepsilon A^{2}}{2}=1+\frac{q_{0} \varepsilon A^{2}}{4}+\frac{3\left(1-q_{0}\right) \varepsilon A^{2}}{4},
$$

which is an amplitude equation to determine $A$ as follows:

$$
A= \pm \frac{2}{\sqrt{3}} \sqrt{1-\frac{1}{\varepsilon}}
$$

The accuracy of $A$ is of the first-order, which coincides to that derived by Liao (2004) using the homotopy analysis method at the first-order.

Inserting Eqs. (13) and (30) into Eq. (27) with $k=2$, yields

$$
y_{2}^{\prime \prime}(x)+y_{2}(x)=\frac{\varepsilon A^{2}}{32}\left(\alpha_{1}+2 e_{0} \cos 2 x\right)(\sin x+\sin 3 x)+\alpha_{2} \sin x .
$$

To avoid the secular solution, we can take

$$
\alpha_{2}=-\frac{\varepsilon A^{2} \alpha_{1}}{32}=\frac{\left(3-2 q_{0}\right) \varepsilon^{2} A^{4}}{128} .
$$




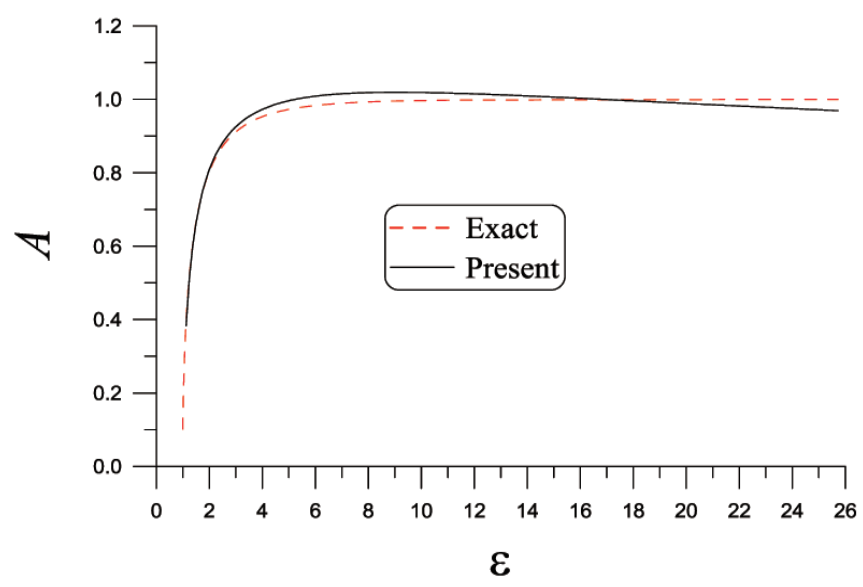

Figure 1. For the Duffing equation, comparing $A$ of exact solution and the solution obtained by the present method

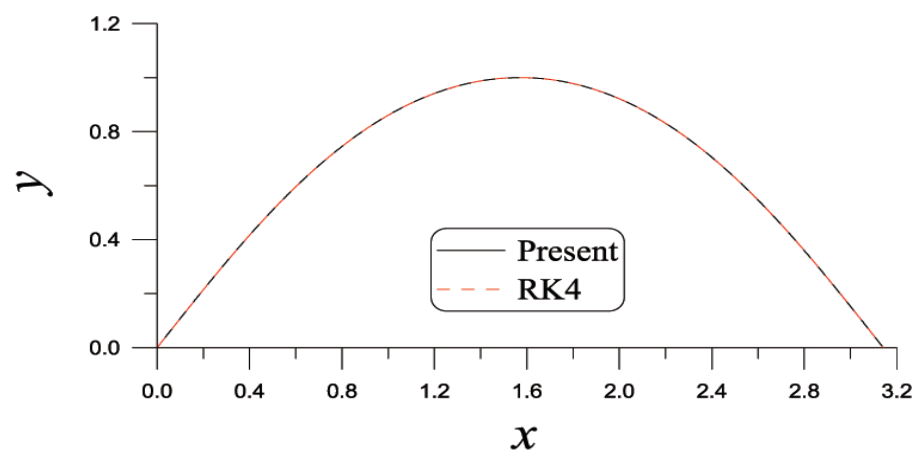

Figure 2. For the Duffing equation, comparing exact solution $y$ obtained by the Runge-Kutta method (RK4) and the solution obtained by the present method

After inserting Eq. (34) for $\alpha_{2}$ into Eq. (33) the second-order solution is found to be

$$
y_{2}(x)=\frac{\left(3 e_{1}-2 e_{0}\right) \varepsilon A^{2}}{256}(\sin x+\sin 3 x)+\frac{e_{0} \varepsilon A^{2}}{768}(\sin x-\sin 5 x),
$$

where the two terms of $\sin x$ are appeared for satisfying $y_{2}(\pi / 2)=0$.

Inserting $p=1$ into Eq. (24) with $m=2$ and with the aid of Eqs. (19), (29) and (34), it follows a second-order amplitude equation:

$$
\begin{aligned}
& \varepsilon-\frac{q_{0} \varepsilon A^{2}}{2}=1+\frac{q_{0} \varepsilon A^{2}}{4}+\frac{3\left(1-q_{0}\right) \varepsilon A^{2}}{4}-\frac{\left(3-2 q_{0}\right) \varepsilon^{2} A^{4}}{128} \Rightarrow \frac{3 \varepsilon A^{2}}{4}+\frac{\left(2 q_{0}-3\right) \varepsilon^{2} A^{4}}{128}+1-\varepsilon=0, \\
& b_{0}:=\frac{96}{\left(2 q_{0}-3\right) \varepsilon}, c_{0}:=\frac{128(1-\varepsilon)}{\left(2 q_{0}-3\right) \varepsilon^{2}}, A= \pm \frac{1}{\sqrt{2}} \sqrt{\sqrt{b_{0}^{2}-4 c_{0}}-b_{0}} .
\end{aligned}
$$

The accuracy of $A$ is up to second-order. When $\varepsilon=1, A=0$ and Eqs. (7) and (8) have a solution $y=\sin x$. As $\varepsilon<1$, there exists no solution. Therefore, $\varepsilon=1$ is a bifurcation point.

We fix $q_{0}=2.225$ and compare the exact solution (9) to that computed by Eq. (37). As shown in Figure 1, these two curves are close. As shown in Figure 2, the solution obtained from Eq. (23) with $p=1, m=2, q_{0}=3$ and $\varepsilon=1.5$ is close to the one obtained by using the fourth-order Runge-Kutta method (RK4) to integrate Eq. (7), where the error is smaller than $2.34 \times 10^{-4}$. 
To enhance the accuracy, we can raise the order to three. Inserting Eqs. (13), (30) and (35) into Eq. (27) with $k=3$, yields

$$
\begin{aligned}
& y_{3}^{\prime \prime}(x)+y_{3}(x)=\left(\alpha_{1}+2 e_{0} \cos 2 x\right) y_{2}(x)+\alpha_{2} y_{1}(x)+\alpha_{3} y_{0}(x)=\left[\frac{\alpha_{2} \varepsilon A^{2}}{32}-\frac{\left(2 e_{0}-3 e_{1}\right)^{2} \varepsilon A^{2}}{256}\right] \sin 3 x \\
& +\left[\frac{e_{0}\left(3 e_{1}-2 e_{0}\right) \varepsilon A^{2}}{256}-\frac{\alpha_{1} e_{0} \varepsilon A^{2}}{768}\right] \sin 5 x-\frac{e_{0}^{2} \varepsilon A^{2}}{768} \sin 7 x \\
& +\left[\alpha_{3}-\frac{e_{0}^{2} \varepsilon A^{2}}{768}+\frac{\alpha_{2} \varepsilon A^{2}}{32}-\frac{\alpha_{1}\left(2 e_{0}-3 e_{1}\right) \varepsilon A^{2}}{256}+\frac{\alpha_{1} e_{0} \varepsilon A^{2}}{768}\right] \sin x .
\end{aligned}
$$

We can take

$$
\alpha_{3}=\frac{e_{0}^{2} \varepsilon A^{2}}{768}-\frac{\alpha_{2} \varepsilon A^{2}}{32}+\frac{\alpha_{1}\left(2 e_{0}-3 e_{1}\right) \varepsilon A^{2}}{256}-\frac{\alpha_{1} e_{0} \varepsilon A^{2}}{768}=\frac{\left(3 q_{0}^{2}-8 q_{0}+6\right) \varepsilon A^{2}}{4096},
$$

and the solution of Eq. (38) is found to be

$$
\begin{aligned}
& y_{3}(x)=\frac{1}{8}\left[\frac{\left(2 e_{0}-3 e_{1}\right)^{2} \varepsilon A^{2}}{256}-\frac{\alpha_{2} \varepsilon A^{2}}{32}\right](\sin 3 x+\sin x) \\
& +\frac{1}{24}\left[\frac{\alpha_{1} e_{0} \varepsilon A^{2}}{768}-\frac{e_{0}\left(3 e_{1}-2 e_{0}\right) \varepsilon A^{2}}{256}\right](\sin 5 x-\sin x)+\frac{e_{0}^{2} \varepsilon A^{2}}{36864}(\sin 7 x+\sin x),
\end{aligned}
$$

where the three terms of $\sin x$ are appeared to render $y_{3}(\pi / 2)=0$. The amplitude $A$ is solved from the following amplitude equation:

$$
\frac{3 q_{0}^{2}-8 q_{0}+6}{4096}\left(\varepsilon A^{2}\right)^{3}-\frac{2 q_{0}-3}{128}\left(\varepsilon A^{2}\right)^{2}-\frac{3 \varepsilon A^{2}}{4}+\varepsilon-1=0,
$$

which can be obtained by using the Newton iteration method in Eq. (12) very fast.

The analytic solution up to third-order is obtained from Eq. (23) with $p=1$ and $m=3$ :

$$
\begin{aligned}
& y(x)=\sin x+\frac{\varepsilon A^{2}}{32}(\sin x+\sin 3 x)+\frac{\left(3 e_{1}-2 e_{0}\right) \varepsilon A^{2}}{256}(\sin x+\sin 3 x)+\frac{e_{0} \varepsilon A^{2}}{768}(\sin x-\sin 5 x) \\
& +\frac{1}{8}\left[\frac{\left(2 e_{0}-3 e_{1}\right)^{2} \varepsilon A^{2}}{256}-\frac{\alpha_{2} \varepsilon A^{2}}{32}\right](\sin x+\sin 3 x) \\
& +\frac{1}{24}\left[\frac{\alpha_{1} e_{0} \varepsilon A^{2}}{768}-\frac{e_{0}\left(3 e_{1}-2 e_{0}\right) \varepsilon A^{2}}{256}\right](\sin 5 x-\sin x)+\frac{e_{0}^{2} \varepsilon A^{2}}{36864}(\sin x+\sin 7 x) .
\end{aligned}
$$

We compare it with different $\varepsilon$ to that computed by the RK4 applied to Eq. (7) within an interval $x \in[0, \pi]$. The values of $q_{0}$ are listed in the table. We also list $A$ obtained from Eq. (41) in Table 1. We can observe that even for large values of $\varepsilon$ and $A$ the presented method is very accurate.

Table 1. Comparing the maximum error (ME) for the analytic eigenfunction of Duffing equation with different $\varepsilon$ obtained by the present method and the RK4, and listing $A$

\begin{tabular}{|l|l|l|l|l|l|l|}
\hline$\varepsilon$ & 1 & 1.2 & 1.4 & 1.6 & 2 & 2.5 \\
\hline$q_{0}$ & 3.04 & 3.04 & 3.1 & 3.15 & 3.15 & 3.5 \\
\hline$A$ & 0 & 0.4695158 & 0.6121567 & 0.6987549 & 0.8016252 & 0.8722130 \\
\hline $\mathrm{ME}$ & $1.59 \times 10^{-13}$ & $8.64 \times 10^{-6}$ & $1.27 \times 10^{-4}$ & $2.67 \times 10^{-4}$ & $1.36 \times 10^{-2}$ & $1.92 \times 10^{-2}$ \\
\hline
\end{tabular}

\section{An Unconventional Periodic Problem}

We consider an unconventional periodic problem of Duffing equation:

$$
\begin{aligned}
& \ddot{w}(t)+w(t)-\beta w^{3}(t)=0, \\
& w(0)=w(H)=0,
\end{aligned}
$$

where $H=T / 2$ is one half of an unknown period $T$ of the free Duffing oscillator with the zero initial displacement. In order to determine $T$, we give an extra condition:

$$
w(H / 2)=A,
$$


which is a given displacement at one quarter of the period $H / 2=T / 4$ with $A$ a nonzero constant. Because $w(0)=0$ and $\dot{w}(0)$ is not given, Eqs. (43)-(45) constitute an unconventional periodic problem of the Duffing equation, of which we want to determine the period $T$ and the corresponding periodic solution.

Let

Eqs. (43)-(45) become

$$
x:=\frac{\pi t}{H}, \varepsilon=\frac{H^{2}}{\pi^{2}}, v(x)=w(t)
$$

$$
\begin{aligned}
& v^{\prime \prime}(x)+\varepsilon\left[v(x)-\beta v^{3}(x)\right]=0, \\
& v(0)=0, v(\pi / 2)=A, v(\pi)=0
\end{aligned}
$$

Now, letting

$$
y(x):=\frac{v(x)}{A} \Rightarrow y(\pi / 2)=\frac{v(\pi / 2)}{A}=1,
$$

Eqs. (47) and (48) are recast to

$$
\begin{aligned}
& y^{\prime \prime}(x)+\varepsilon\left[y(x)-\beta A^{2} y^{3}(x)\right]=0, \\
& y(0)=0, y(\pi / 2)=1, y(\pi)=0 .
\end{aligned}
$$

Making a linearization of Eq. (50) around $y_{0}(x)=\sin x$, we come to Eq. (18) again, but with

$$
\lambda=\varepsilon-\frac{q_{0} \beta \varepsilon A^{2}}{2}, e_{0}=-\frac{q_{0} \beta \varepsilon A^{2}}{4}, e_{1}=\frac{\left(1-q_{0}\right) \beta \varepsilon A^{2}}{4}=e_{0}+\frac{\beta \varepsilon A^{2}}{4} .
$$

At the same time, $\alpha_{1}$ and $\alpha_{2}$ in Eqs. (29) and (34) are changed to

$$
\alpha_{1}=\frac{\left(2 q_{0}-3\right) \beta \varepsilon A^{2}}{4}, \alpha_{2}=\frac{\left(3-2 q_{0}\right) \beta^{2} \varepsilon^{2} A^{4}}{128} .
$$

Inserting $p=1$ into Eq. (24) with $m=2$ and with the aid of Eqs. (52) and (53), it follows that

$$
\begin{aligned}
& \frac{\left(2 q_{0}-3\right) \beta^{2} A^{4}}{128} \varepsilon^{2}+\frac{3 \beta A^{2}-4}{4} \varepsilon+1=0, \\
& a_{0}:=\frac{\left(2 q_{0}-3\right) \beta^{2} A^{4}}{128}, b_{0}:=\frac{3 \beta A^{2}-4}{4}, \varepsilon=-\frac{\sqrt{b_{0}^{2}-4 a_{0}}+b_{0}}{2 a_{0}} .
\end{aligned}
$$

Then, the period $T$ is determined by

$$
T=2 \pi \sqrt{\varepsilon}=2 \pi \sqrt{\frac{-\sqrt{b_{0}^{2}-4 a_{0}}-b_{0}}{2 a_{0}}},
$$

which is obtained from $T=2 H, H^{2}=\pi^{2} \varepsilon$ in Eq. (46), and $\varepsilon$ in Eq. (55). The second-order solution is given by

$$
\begin{aligned}
& w(t)=A \sin \frac{2 \pi t}{T}+\frac{\beta \varepsilon A^{3}}{32}\left(\sin \frac{2 \pi t}{T}+\sin \frac{6 \pi t}{T}\right) \\
& +\frac{\left(3 e_{1}-2 e_{0}\right) \beta \varepsilon A^{3}}{256}\left(\sin \frac{2 \pi t}{T}+\sin \frac{6 \pi t}{T}\right)+\frac{e_{0} \beta \varepsilon A^{3}}{768}\left(\sin \frac{2 \pi t}{T}-\sin \frac{10 \pi t}{T}\right) .
\end{aligned}
$$

As shown in Figure 3, the solution in Eq. (57) is close to the one obtained by using the RK4 to integrate Eq. (43) with $\beta=-0.2$ and $A=2$, where the error is smaller than $1.75 \times 10^{-4}$.

The accuracy of $T$ in Eq. (56) is compared to the exact one:

$$
T_{e x}=4 \sqrt{2} \int_{0}^{\pi / 2} \frac{d \theta}{\sqrt{2-\beta A^{2}\left(1+\sin ^{2} \theta\right)}},
$$

whose applicable range is $\beta A^{2}<1$. For Eq. (56), the applicable range is $\beta A^{2}<4 / 3$. Upon fixing $A=1$ and $q_{0}=3$, we compare $T$ obtained from Eqs. (56) and (58) in Table 2. For the analytic solutions in Eq. (57) with different value of $\beta$, we 

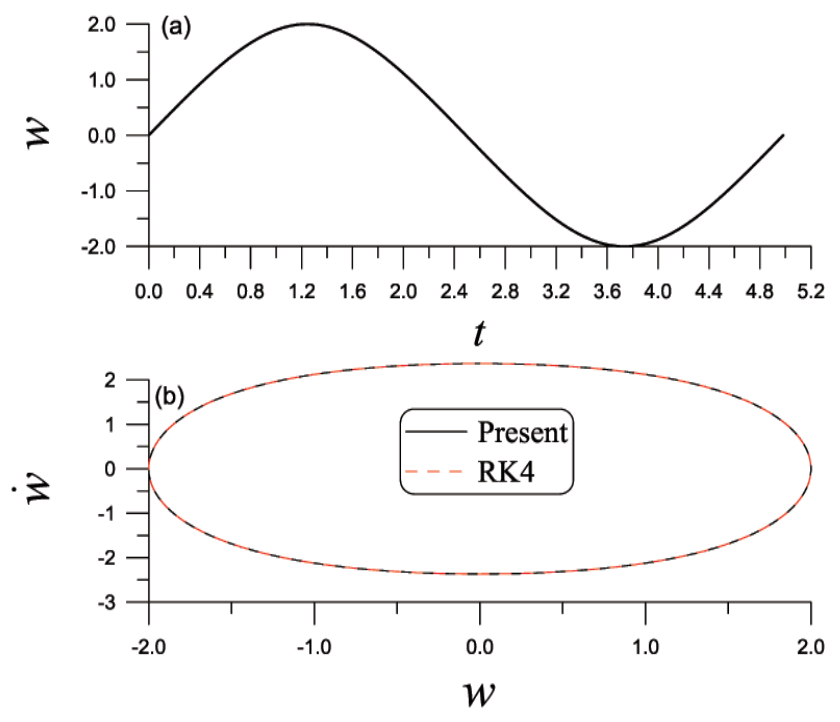

Figure 3. For the unconventional periodic problem of Duffing equation, comparing exact solution $w(t)$ obtained by the Runge-Kutta method (RK4) and the solution obtained by the present method

compare them with that computed by the RK4 within one period $T$. Again, we can appreciate the high-accuracy of the presented method.

Table 2. Comparing the maximum error (ME) for the analytic solutions of Duffing oscillator with different $\beta$, obtained by the present method and the RK4, and comparing the periods

\begin{tabular}{|l|l|l|l|l|l|l|}
\hline$\beta$ & -0.8 & -0.5 & -0.2 & 0.1 & 0.2 & 0.5 \\
\hline ME & $8.77 \times 10^{-5}$ & $2.61 \times 10^{-5}$ & $2.61 \times 10^{-6}$ & $6.59 \times 10^{-7}$ & $7.73 \times 10^{-6}$ & $1.23 \times 10^{-3}$ \\
\hline Exact $T$ & 4.981972 & 5.366659 & 5.861179 & 6.533840 & 6.815901 & 8.008619 \\
\hline Eq. (56) & 4.981998 & 5.366667 & 5.861180 & 6.533840 & 6.815903 & 8.008901 \\
\hline
\end{tabular}

\section{Conclusions}

To simplify the homotopy perturbation method, we have proposed a new analytic method based on a linearization technique being executed on the nonlinear Duffing equation and then applied He's homotopy perturbation method to solve two nonlinear problems of Duffing equations in space and in time. The first problem is the bifurcation of Duffing equation in space, where a critical parameter $\varepsilon=1$ initiates the bifurcation to the existence of non-trivial solutions. The second problem is an unconventional periodic problem of Duffing equation in time. By using the derived equations we can determine the period and analytic periodic solutions very accurately. The presented linearized homotopy perturbation method is easily carried out with merely $k$ linear terms appeared in the right side for each $k$ th order linear differential equation. Comparing to the original He's homotopy perturbation method, the linearized homotopy perturbation method can save much algebraic computations.

Although we were restricted ourselves to the two nonlinear problems of the Duffing equations in space and in time, the presented linearized homotopy methods are easily extended to other nonlinear problems governed by other nonlinear second-order differential equations. For example, the bifurcation problem of other nonlinear strut in an one-dimensional space can be treated by the presented method, and the unconventional periodic problem of nonlinear second-order differential equations can be studied by the presented linearized homotopy methods, whose results may be more accurate than that using the conventional conditions with initial displacement and velocity and with other analytic methods.

\section{References}

Bujurke, N. M., Salimath, C. S., \& Shiralashetti, S. C. (2008). Computation of eigenvalues and solutions of regular Sturm-Liouville problems using Haar wavelets. J. Comput. Appl. Math., 219, 90-101. https://doi.org/10.1016/j.cam.2007.07.005

Cartmell, M. (1990). Introduction to Linear, Parametric and Nonlinear Vibrations. Chapman and Hall, New York. 
Chu, H. P., \& Lo, C. Y. (2011). Application of the differential transform method for solving periodic solutions of strongly non-linear oscillators. Comput. Model. Eng. Sci., 77, 161-172.

Donescu, P., Virgin, L. N., \& Wu, J. J. (1996). Periodic solutions of an unsymmetric oscillator including a comprehensive study of their stability characteristics. J. Sound Vib., 192, 959-976. https://doi.org/10.1006/jsvi.1996.0228

Gadella, M., Giacomini, H., \& Lara, L. P. (2015). Periodic analytic approximate solutions for the Mathieu equation. Appl. Math. Comput., 271, 436-445. https://doi.org/10.1016/j.amc.2015.09.018

He, J. H. (1999). Variational iteration method - a kind of non-linear analytical technique: some examples. Int. J. Nonlinear Mech., 34, 699-708. https://doi.org/10.1016/S0020-7462(98)00048-1

He, J. H. (2000a). Variational iteration method for autonomous ordinary systems. Appl. Math. Comput., 114, 115-123. https://doi.org/10.1016/S0096-3003(99)00104-6

He, J. H. (2000b). A coupling method of a homotopy technique and a perturbation technique for non-linear problems. Int. J. Non-linear Mech., 35, 37-43. https://doi.org/10.1016/S0020-7462(98)00085-7

He, J. H. (2001). Bookkeeping parameter in perturbation methods. Int. J. Non-linear Sci. Numer. Simul., 2, 257-264. https://doi.org/10.1515/IJNSNS.2001.2.3.257

He, J. H. (2002). Modified Lindstedt-Poincare methods for some strongly nonlinear oscillations Part I: expansion of a constant. Int. J. Non-linear Mech., 37, 309-314. https://doi.org/10.1016/S0020-7462(00)00116-5

He, J. H. (2003). Homotopy perturbation method: a new nonlinear analytical technique. Appl. Math. Comput., 135, 73-79. https://doi.org/10.1016/S0096-3003(01)00312-5

He, J. H. (2005). Homotopy perturbation method for bifurcation of nonlinear problems. Int. J. Non-linear Sci. Numer. Simul., 6, 207-208. https://doi.org/10.1515/IJNSNS.2005.6.2.207

He, J. H. (2006). Some asymptotic methods for strongly nonlinear equations. Int. J. Modern Phys. B, 20, 1141-1199. https://doi.org/10.1142/S0217979206033796

He, J. H., \& Abdou, A. (2007). New periodic solutions for nonlinear evolution equations using exp-function method. Chaos Soliton Fract., 34, 1421-1429. https://doi.org/10.1016/j.chaos.2006.05.072

He, J. H., \& El-Dib, Y. O. (2020). Homotopy perturbation method for Fangzhu oscillator. J. Math. Chem., 58, 2245-2253. https://doi.org/10.1007/s10910-020-01167-6

He, J. H., \& El-Dib, Y. O. (2021). Homotopy perturbation method with three expansions. J. Math. Chem., 59, 1139-1150. https://doi.org/10.1007/s10910-021-01237-3

Herisanu, N., \& Marinca, V. (2010). A modified variational iteration method for strongly nonlinear problems. Nonlinear Sci. Lett. A, 1, 183-192.

Kahn, P. B., \& Zarmi, Y. (1998). Nonlinear Dynamics: Exploration through Normal Forms. John Wiley \& Sons, New York.

Khan, N. A., Liu, C.-S., \& Riaz, F. (2016). An optimally scaled polynomial-Fourier-series method for the numerical solution of the Duffing oscillator. Int. J. Appl. Nonl. Sci., 2(5), 290-310. https://doi.org/10.1504/IJANS.2016.085806

Koroglu, C., \& Ozis, T. (2011). Applications of parameter-expanding method to nonlinear oscillators in which the restoring force is inversely proportional to the dependent variable or in form of rational function of dependent variable. Comput. Model Eng. Sci., 75, 223-234.

Liao, S. J. (2004). Beyond Perturbation: Introduction to the Homotopy Analysis Method. Chapman \& Hall/CRC, New York.

Liu, C.-S. (2020). Analytic solutions of the eigenvalues of Mathieu's equation. J. Math. Research, 12(1), 1-11. https://doi.org/10.5539/jmr.v12n1p1

Liu, L., Thomas, J. P., Dowell, E. H., Attar, P., \& Hall, K. C. (2006). A comparison of classical and high dimension harmonic balance approaches for a Duffing oscillator. J. Comput. Phys., 215, 298-320. https://doi.org/10.1016/j.jcp.2005.10.026

Motsa, S. S., \& Sibanda, P. (2012). A note on the solutions of the Van der Pol and Duffing equations using a linearisation method. Math. Prob. Eng., 2012, 693453, 10 pages. https://doi.org/10.1155/2012/693453

Nayfeh, A. H. (1979). Perturbation Methods. Wiley, New York. 
Ozis, T., \& Yildirim, A. (2007). A study of nonlinear oscillators with $u^{1 / 3}$ force by He's variational iteration method. $J$. Sound Vib., 306, 372-376. https://doi.org/10.1016/j.jsv.2007.05.021

Shou, D. H. (2009). The homotopy perturbation method for nonlinear oscillators. Comput. Math. Appl., 58, 2456-2459. https://doi.org/10.1016/j.camwa.2009.03.034

Tao, Z. L. (2009). Frequencyamplitude relationship of nonlinear oscillators by Hes parameter-expanding method. Chaos Soliton Fract., 41, 642-645. https://doi.org/10.1016/j.chaos.2008.02.036

Wu, B. S., Sun, W. P., \& Lim, C. W. (2006). An analytical approximate technique for a class of strongly non-linear oscillators. Int. J. Non-linear Mech., 41, 766-774. https://doi.org/10.1016/j.ijnonlinmec.2006.01.006

Xu, L. (2007). Hes parameter-expanding methods for strongly nonlinear oscillators. J. Comput. Appl. Math., 207, 148-154. https://doi.org/10.1016/j.cam.2006.07.020

\section{Copyrights}

Copyright for this article is retained by the author(s), with first publication rights granted to the journal.

This is an open-access article distributed under the terms and conditions of the Creative Commons Attribution license (http://creativecommons.org/licenses/by/4.0/). 